\title{
Synthesis of selectively fluorinated cyclohexanes: The observation of phenonium rearrangements during deoxyfluorination reactions on cyclohexane rings with a vicinal phenyl substituent.
}

\author{
Tetiana Bykova, Nawaf Al-Maharik, Alexandra M. Z. Slawin, David O’Hagan* \\ School of Chemistry, University of St Andrews, North Haugh, St Andrews, KY16 9ST, UK.
}

\begin{abstract}
A study focused on the synthesis of a derivative of all-cis phenyl-2,3,5,6tetrafluorocyclohexane, incorporating a methyl group at the benzylic position, found that the fluorination reactions of diepoxide and diol precursors, were susceptible to rearrangement and unexpected products. The origin of these rearranged products can be rationalised by aryl migrations occurring via phenonium ion intermediates, in adventitious pathways occurring during deoxofluorination reactions with hydrogen fluoride reagents.
\end{abstract}

We dedicate this paper to Professor Veronique Gouverneur in recognition of her 2015 ACS Award for Creative work in Fluorine Chemistry.

Tel +441334473803 Fax +441334473808Ｅ.mail address do1@st-andrews.ac.uk

Keywords:

Fluoroaliphatics

Phenonium rearrangement

Deoxofluorination reactions

fluorocyclohexanes

All-cis tetrafluorocyclohexane motif

\section{Introduction}

We have recently introduced all-cis phenyl-2,3,5,6-tetrafluorocyclohexane $\mathbf{1}$ as the starting point for the synthesis of a diversity of products $\mathbf{2}$ carrying a tetrafluorocyclohexyl motif with four fluorine up [1-6]. The diversity emerges as the aromatic ring of $\mathbf{1}$ can be modified by a range of standard electrophilic aromatic substitution reactions [4,6]. This gives rise to products 2 that contain a unique motif which has four fluorine atoms on one face of a cyclohexane and offers a facially polarized ring, the properties of which appear unique and remain to be fully explored in medicinal and agrochemical discovery programmes. Compound $\mathbf{1}$ was prepared in a synthesis protocol starting with a Birch reduction of biphenyl 3 to give diene 4 [7-9] followed by diepoxidation with $m$ CPBA to generate diepoxide 5 [4,5]. This diepoxide was then treated with $3 \mathrm{HF} . \mathrm{NEt}_{3}$ and converted to inseparable diols 6 and 7. These diols could also be converted to their corresponding triflates 8 and $\mathbf{9}$. However when the triflates $\mathbf{8}$ and $\mathbf{9}$, which could be separated, were treated individually with $3 \mathrm{HF}^{-N_{E t}}{ }_{3}$, then $\mathbf{8}$ gave $\mathbf{1}$ exclusively, and $\mathbf{9}$ gave cyclohexene $\mathbf{1 0}$ exclusively [5]. The stereochemistry of cyclohexene $\mathbf{1 0}$ indicated that the fluorination had occurred with retention of configuration, consistent with a rearrangement via the symmetrical phenonium ion 11 [5]. This was further supported by a deuterium isotope labelling experiment. 


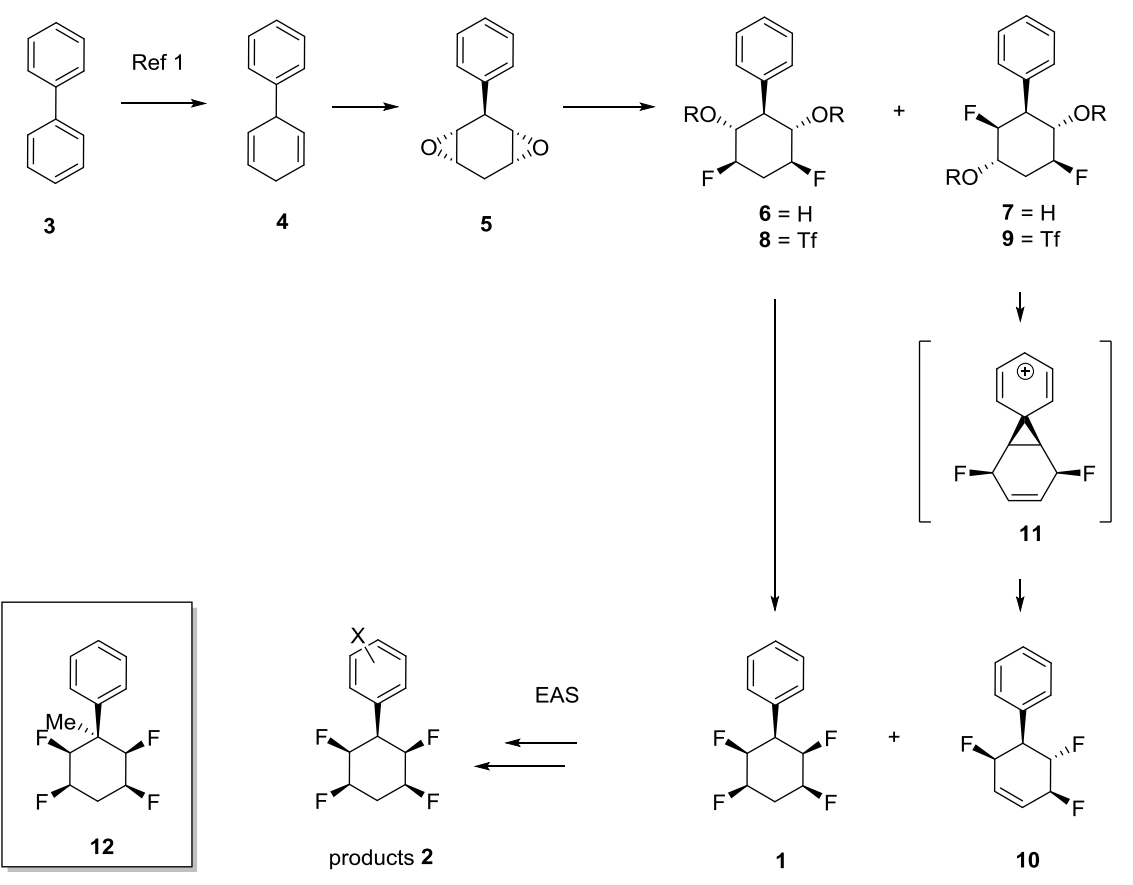

Scheme 1 Previous work [1,5]

As part of a programme to extend the structural diversity of the all-cis tetrafluorocyclohexane motifs $\mathbf{2}$ we were interested in placing a methyl group at the benzylic position to generate products such as 12. To that end we started with the well known modification of the Birch reduction of biphenyl 1 which involves in situ methylation to generate diene 13 [7,9]. It was then required to progress 13 in a similar manner to that previously carried out for $\mathbf{4}$, to generate $\mathbf{1}$. In this paper we report that the transformations with the methyl derivative were prone to aryl migrations consistent again with phenonium intermediates.

\section{Results and discussion}

The Birch reduction of biphenyl $\mathbf{1}$ followed by an in situ methyl iodide quench generated diene 13, a reaction which occurs in almost quantitative yield [7,9]. Epoxidation of $\mathbf{1 3}$ with an excess of $m \mathrm{CPBA}$ was not particularly stereoselective and afforded a mixture of the diepoxide isomers 14, 15 and 16 in the ratio 9.5:1:1.1 respectively, but in favour of diepoxide 14, where the epoxide oxygens are syn to each other but anti to the phenyl group (Scheme 2) [10,11]. Isomer 14 was readily purified by chromatography and was recovered in $68 \%$ yield and its structure and stereochemistry was confirmed by X-ray crystallography as shown in Figure 1. This was the only isomer progressed in the subsequent fluorination studies.

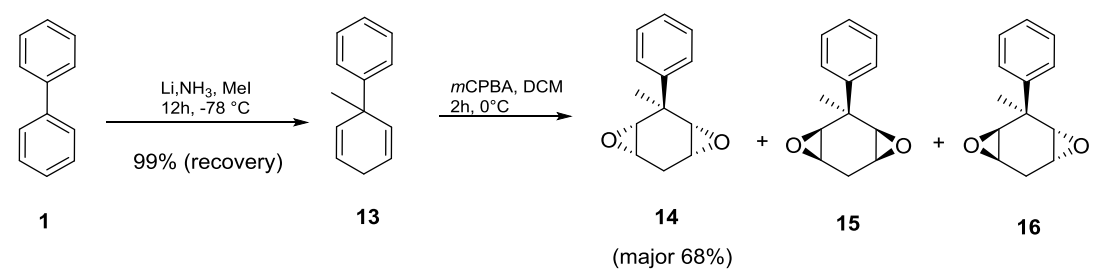

Scheme 2 Synthesis of diepoxide diastereisomers 14, 15 and 16. 


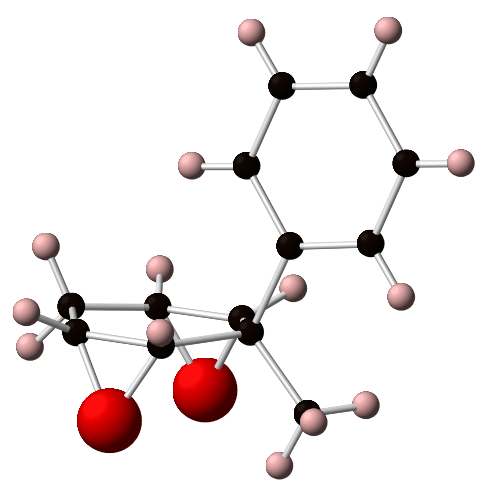

Fig 1: X-Ray structure of diepoxide 14. Crystallographic data (excluding structure factors) for the structures in this paper have been deposited with the Cambridge Crystallographic Data Centre (CCDC 1409214) [12].

Ring opening of diepoxide 14 was firstly explored with pyridine.HF [13,14]. This resulted in an efficient conversion but to the rather unexpected difluorodiol 18. The structure and stereochemistry of product 18 was also confirmed by X-ray structure analysis as shown in Figure 2. In retrospect we can rationalize a rearrangement reaction, which most reasonably proceeded via a phenonium type intermediate as represented by 17 in Scheme 3 [5,15-20]. This is an interesting outcome but not particularly useful for the preparation of a tetrafluorocyclohexyl motif.

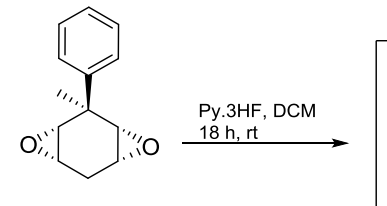

14

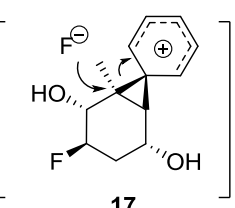

17

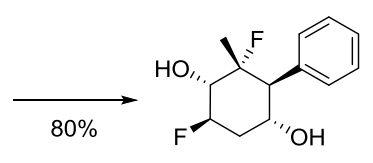

18

Scheme 3: Synthesis protocol and X-ray structure of difluoro diol 18.

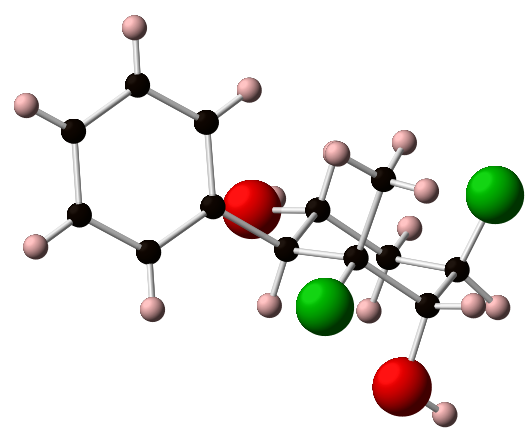


Fig 2: X- Ray structure of difluorodiol 18. Crystallographic data (excluding structure factors) for the structures in this paper have been deposited with the Cambridge Crystallographic Data Centre (CCDC 1409215) [12].

Treatment of dipoxide 14 with the more basic $\mathrm{Et}_{3} \mathrm{~N}$.3HF reagent, gave a more predictable outcome and this reaction furnished a mixture of inseparable difluoro diols $\mathbf{1 9}$ and $\mathbf{2 0}$ in 2.8:1 ratio as illustrated in Scheme 4 [4,5,21]. Under the latter conditions rearranged product 18 was not detected. This outcome clearly illustrates how the increased acidity of pyridine.HF relative to $\mathrm{Et}_{3} \mathrm{~N}$. $3 \mathrm{HF}$ can give rise to very different product outcomes. Diols 19 and 20 were then converted to the corresponding triflates $\mathbf{2 1}$ and $\mathbf{2 2}$ [5]. These triflates were not easily separable and were therefore treated together with $\mathrm{Et}_{3} \mathrm{~N} \cdot 3 \mathrm{HF}$ at $110^{\circ} \mathrm{C}$.
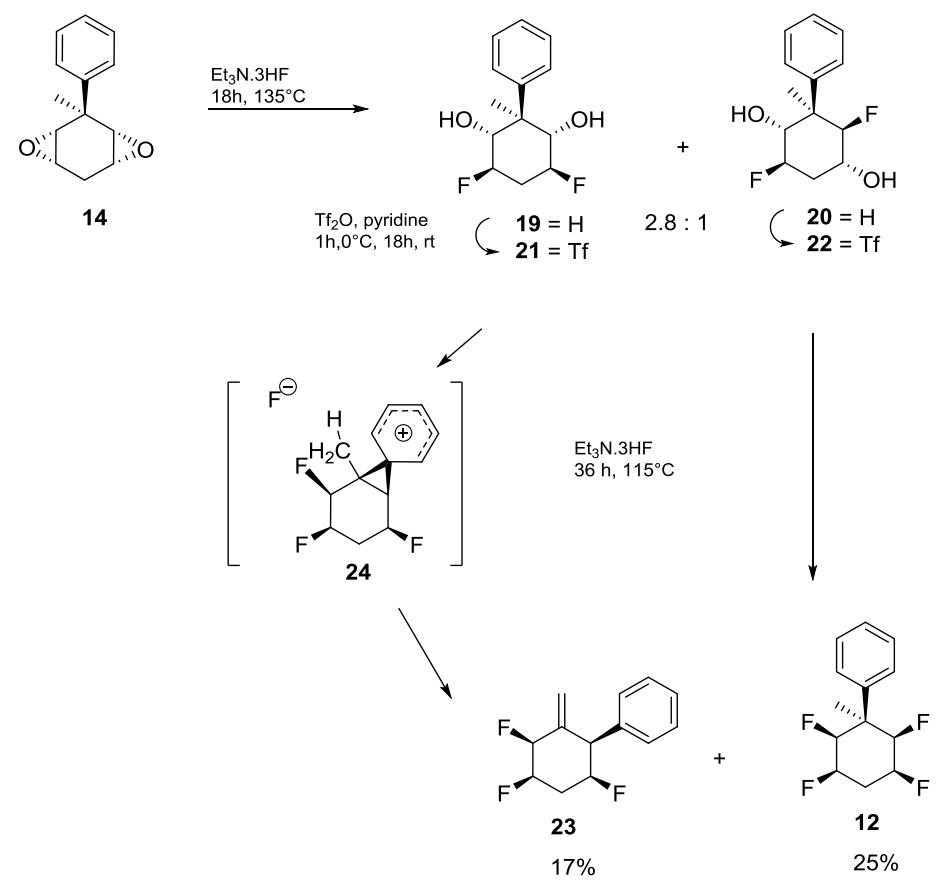

Scheme 4: Synthesis of fluorinated cyclohexanes 23 and 12.

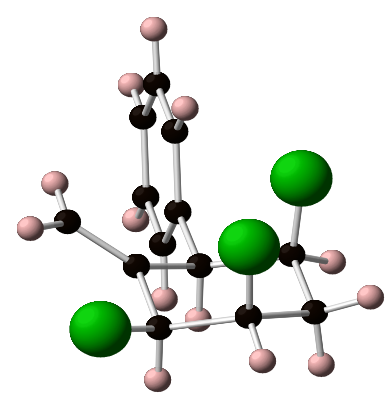

23

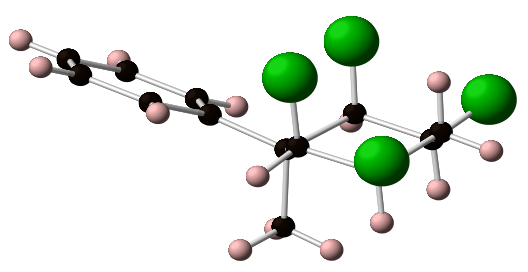

12 
Fig 3: X-ray structures of products 23 and 12. Crystallographic data (excluding structure factors) for the structures in this paper have been deposited with the Cambridge Crystallographic Data Centre (CCDC 1409216 for 23 and CCDC 1409213 for 12) [12].

On work up this gave two products 23 and 12, which were readily separated by chromatography. Both were crystalline solids and they each proved amenable to X-ray structure analysis as shown in Figure 3. The major product $\mathbf{1 2}$ was the anticipated tetrafluorocyclohexane with a methyl group located at the benzylic position. The structure of $\mathbf{2 3}$, which contains an exo-methylene group, was unanticipated. It can be rationalized retrospectively as arising from an aryl migration most probably progressing via a phenonium ion intermediate such as $\mathbf{2 4}$. This intermediate then collapsed in an E1 type elimination process as illustrated in Scheme 4.

An aryl tetrafluorocyclohexane, with four fluorines all pointing 'up' and positioned at the 2,3,5 and 6 of the cyclohexane ring, presents an attractive option for building this facially polarized ring system into more complex molecular architectures. However we have found in this, and a previous study, that the deoxyfluorination reactions to introduce the last two fluorines of these ring systems is often compromised by the susceptibility of the aryl ring to become involved in neighbouring group participation as the substitution reaction progresses. If this achieves a full phenonium intermediate, then the various breakdown pathways generate a mixture of products.

\section{Conclusion}

In this paper we report some deoxyfluorination reactions of phenylcyclohexane epoxides and report products that arise from the involvement of the aryl ring in neighbouring group participation. The unanticipated reaction modes which compete with more straightforward deoxyfluorination substitutions can be accounted for by breakdown of phenonium ion intermediates.

\section{Experimental}

\subsection{General information}

${ }^{1} \mathrm{H},{ }^{13} \mathrm{C}$ and ${ }^{19} \mathrm{~F}$ NMR spectra were recorded on Bruker Avance or Avance III spectometers at 500 $\mathrm{MHz}\left({ }^{1} \mathrm{H} \mathrm{NMR}\right), 125 \mathrm{MHz}\left({ }^{13} \mathrm{C} \mathrm{NMR}\right)$ and $470 \mathrm{MHz} z\left({ }^{19} \mathrm{~F}\right.$ NMR). The data for chemical shifts were stated as $\delta$ in units of parts per million in relations to residual solvent and were referenced to the internal solvent, where appropriate. Coupling constants were reported in Hertz (Hz). Merck silica gel 60 F254 glass-supported plates has been used for analytical thin layer chromatography (TLC) and observed by absorption of UV light ( $\lambda$ max 253 or $365 \mathrm{~nm}$ ), or by thermal development after dipping in an aqueous solution of potassium permanganate, potassium carbonate and sodium hydroxide. Flash column chromatography was carried out using Merck Geduran silica gel 40-63 micron, under a positive pressure of compressed air with eluting with solvents as supplied. Melting points were recorded on an Electrothermal 9100 melting point apparatus and are uncorrected. Electrospray mass 
spectra were recorded at the University of St Andrews Mass Spectrometry facility on either a Micromass LC TOF spectrometer or a ThermoFisher Orbitrap Excalibur spectrometer from solutions of the analyate in methanol. Additional spectra were obtained at the EPSRC National Mass Spectrometry Service Centre at Swansea using a ThermoFisher Orbitrap LQT XL spectrometer fitted with an ASAP solids probe operating in atmospheric pressure chemical ionisation (APCI) mode.

\section{2. (1R,2s,3S,5R,7S)-2-Methyl-2-phenyl-4,8-dioxatricyclo[5.1.0.0 $\left.0^{3,5}\right]$ octane (14)}

$m$ CPBA (99.58 g, $577 \mathrm{mmol})$ was added to a solution of diene 13 [9] (32.5g, $190 \mathrm{mmol})$ in $\mathrm{CH}_{2} \mathrm{Cl}_{2}$ $(200 \mathrm{~mL})$ at $0{ }^{\circ} \mathrm{C}$. The reaction was allowed to warm to $\mathrm{rt}$ and was stirred for $48 \mathrm{~h}$. The reaction was filtered and the filtrate washed with $10 \%$ aq. $\mathrm{KOH},(100 \mathrm{~mL})$. The aqueous layer was then extracted into dichloromethane $(100 \mathrm{~mL} \times 3)$. The combined organic layers were washed with water $(200 \mathrm{~mL})$, dried. Solvent was removed under reduced pressure. Purification over silica gel (petroleum ether/ethyl acetate 9:1) gave 3 as white crystalline solid, (26.1g, 129mmol, 68\%); M.p. $=86{ }^{\circ} \mathrm{C} ;{ }^{1} \mathrm{H}$ NMR (400 MHz, $\mathrm{CDCl}_{3}$ ) $\delta: 7.48-7.32$ (m, 5H, H-2',3',4'), 3.31 (ddd, $J=4.1,2.8,1.3 \mathrm{~Hz}, 2 \mathrm{H}, \mathrm{H}-2$ ), 3.00-2.96 (m, 2H, H-3), 2.95-2.87 (m, 1H, H-4a), 2.42 (dt, J = 17.3, $2.9 \mathrm{~Hz}, 1 \mathrm{H}, \mathrm{H}-4 \mathrm{~b}), 1.81$ (s, 3H, $\mathrm{CH}_{3}$ ); ${ }^{13} \mathrm{C}$ NMR (101 MHz, $\mathrm{CDCl}_{3}$ ) $\delta: 142.2$ (C-1'), 129.0 (C-3'), 127.6 (C-4'), 126.6 (C-2'), 58.2 (C-2), $51.4(\mathrm{C}-3), 38.3(\mathrm{C}-1), 23.3(\mathrm{C}-4), 19.8\left(\mathrm{CH}_{3}\right)$; FTMS $\left(\mathrm{ESI}^{+}\right) \mathrm{m} / z$ calcd for $([\mathrm{M}]+\mathrm{Na})$ 225.08915; found 225.088

\section{3 (1S,2R,3S,4R,6R)-2,6-Difluoro-2-methyl-3-phenylcyclohexane-1,4-diol (18)}

Pyridine.HF (0.982 $\mathrm{mL}, 1.96 \mathrm{mmol})$ was added to a solution of diepoxide 14 (2 g, $9.84 \mathrm{mmol})$ in dichloromethane $(5 \mathrm{~mL})$ at $-78^{\circ} \mathrm{C}$ under an argon atmosphere. After stirring for $12 \mathrm{~h}$ at $\mathrm{rt}$, aq. $\mathrm{NaHCO}_{3}(10 \%, 30 \mathrm{~mL})$ was added and the organics were extracted into ethyl acetate (40 mL x 3). The combined organic layers were then washed and dried. The solvent was removed under reduced pressure and the residue re-dissolved in a minimum amount of dichloromethane and filtered through silica (petroleum ether/ethyl acetate 7:3). This gave $\mathbf{1 8}$ as a yellow crystalline solid (1.9 $\mathrm{g}, 7.85$ mmol, 80\%); M.p.= $146{ }^{\circ} \mathrm{C}$; ${ }^{1} \mathrm{H}$ NMR (400 MHz, $\mathrm{CDCl}_{3}$ ) $\delta: 7.49-7.27$ (m, 5H, H-2', 3', 4'), 5.02 (ddt, $J=45.7,6.0,3.1 \mathrm{~Hz}, 1 \mathrm{H}, \mathrm{H}-5), 4.37$ (td, $J=11.0,4.6 \mathrm{~Hz}, 1 \mathrm{H}, \mathrm{H}-3), 4.09$ (m, 1H, H-6), 3.42 (dd, $J=13.3,10.9 \mathrm{~Hz}, 1 \mathrm{H}, \mathrm{H}-2), 2.45$ (m, 1H, H-4a), 2.12 (m, 1H, H-4b), 1.27 (dd, 25.3, 3.2 Hz, 3H, $\left.\mathrm{CH}_{3}\right) ;{ }^{13} \mathrm{C}$ NMR (101 MHz, $\mathrm{CDCl}_{3}$ ) $\delta: 135.1$ (C-1'), 130.1 (C-3'), 128.7 (C-2'), 127.7 (C-4'), 89.6 $(\mathrm{dd}, J=169.5,5.3 \mathrm{~Hz}, \mathrm{C}-5), 72.7$ (dd, $J=27.5,19.2 \mathrm{~Hz}, \mathrm{C}-6), 65.7$ (d, $J=10.0 \mathrm{~Hz}, \mathrm{C}-3), 53.9$ (d, $J=$ $18.8 \mathrm{~Hz}, \mathrm{C}-2), 34.0(\mathrm{~d}, J=19.3 \mathrm{~Hz}, \mathrm{C}-4), 20.0\left(\mathrm{dd}, J=23.9,7.2 \mathrm{~Hz}, \mathrm{CH}_{3}\right) ;{ }^{19} \mathrm{~F} \mathrm{NMR}(376 \mathrm{MHz}$, $\mathrm{CDCl}_{3}$ ) $\delta:-143.8$ (m, F-1), -186.9 (m, F-5); ${ }^{19} \mathrm{~F}$ NMR (decoupled) (376 $\left.\mathrm{MHz}, \mathrm{CDCl}_{3}\right) \delta:-143.8$ (F1), -186.9 (F-5); FTMS $\left(\mathrm{ESI}^{+}\right) \mathrm{m} / \mathrm{z}$ calcd for $([\mathrm{M}]+\mathrm{Na})^{+}$265.252; found 265.1009

\subsection{Difluoro-methyl-phenylcyclohexane-1,3-diols (19 and 20)}

$\mathrm{Et}_{3} \mathrm{~N} .3 \mathrm{HF}(6.5 \mathrm{~mL}, 39.6 \mathrm{mmol})$ was added to diepoxide $14(1 \mathrm{~g}, 4.96 \mathrm{mmol})$ at room temperature and under an argon atmosphere. After stirring at $135^{\circ} \mathrm{C}$ for $48 \mathrm{~h}$, the mixture was cooled to room temperature, and aq. $\mathrm{NaHCO}_{3}(10 \%, 200 \mathrm{~mL})$ was added and the product extracted into ethyl acetate (15 mL x 3). The combined organic extracts were dried and concentrated under reduced pressure. The product was passed through a short silica gel pad (petroleum ether/dichloromethane 1:1) to yield a mixture of $\mathbf{1 9}$ and $\mathbf{2 0}$ (1.22 g), which was taken to the next stage without purification. 
A sample of each isomer was purified over silica gel (petroleum ether: diethyl ether / 8:2) for analysis.

\section{$4.5(1 R, 2 s, 3 S, 4 S, 6 R)-4,6-D i f l u o r o-2-m e t h y l-2-p h e n y l c y c l o h e x a n e-1,3-d i o l$ (19)}

M.p. $=128-129{ }^{\circ} \mathrm{C} ;{ }^{1} \mathrm{H}$ NMR (400 MHz, $\left.\mathrm{CDCl}_{3}\right) \delta:$ 7.59-7.38 (m, 5H, H-2', 3', 4'), 4.74 (dddd, $J=$ 49.1, 11.7, 8.9, $5.5 \mathrm{~Hz}, 2 \mathrm{H}, \mathrm{H}-3$ ), 4.04 (dd, $J=13.2,9.1 \mathrm{~Hz}, 2 \mathrm{H}, \mathrm{H}-2), 2.78$ (ddd, $J=11.7,6.3,2.9$ $\mathrm{Hz}, 1 \mathrm{H}, \mathrm{H}-4 \mathrm{a}), 2.00(\mathrm{q}, J=12.1 \mathrm{~Hz}, 1 \mathrm{H}, \mathrm{H}-4 \mathrm{~b}), 1.36\left(\mathrm{~s}, 3 \mathrm{H}, \mathrm{CH}_{3}\right) ;{ }^{13} \mathrm{C} \mathrm{NMR}\left(75 \mathrm{MHz}, \mathrm{CDCl}_{3}\right) \delta$ : 142.1 (C-1'), 129.0 (C-3'), 127.4 (C-4'), 126.4 (C-2'), 90.2 (dd, $J=175.3,15.8$ Hz, C-3), 77.3 (dd, $J$ $=19.71,2.58 \mathrm{~Hz}, \mathrm{C}-2), 47.6(\mathrm{C}-1), 33.2(\mathrm{t}, J=20.7 \mathrm{~Hz}, \mathrm{C}-4), 11.7\left(\mathrm{CH}_{3}\right) ;{ }^{19} \mathrm{~F}$ NMR $(376 \mathrm{MHz}$, $\left.\mathrm{CDCl}_{3}\right) \delta:-188.7(\mathrm{dt}, J=50.3,12.6 \mathrm{~Hz}, \mathrm{~F}-3) ;{ }^{19} \mathrm{~F}$ NMR (decoupled) (376 MHz, $\left.\mathrm{CDCl}_{3}\right) \delta:-188.7(\mathrm{~F}-$ 3); TOF MS $\left(\mathrm{EI}^{+}\right) \mathrm{m} / z$ calcd for $([\mathrm{M}])^{+} 242.1118$; found 242.1120

\section{6 (1R,2R,3R,4R,6R)-3,6-Difluoro-2-methyl-2-phenylcyclohexane-1,4-diol (20)}

Yellow oil; ${ }^{1} \mathrm{H}$ NMR (400 MHz, $\mathrm{CDCl}_{3}$ ) $\delta$ : 7.58-7.36 (m, 5H, 1H, H-2', 3', 4'), 5.08 (dddd, $J=50.9$, 11.1, 8.6, 4.9 Hz, 1H, H-5), $4.69-4.51$ (m, 2H, 1H, H-2, H-6), 4.41 (dt, J = 8.0, 3.7 Hz, 1H, H-5), 2.36 (m, 1H, H-4a), 2.19 (m, 1H, H-4b), 1.56 (d, $\left.J=1.8 \mathrm{~Hz}, 3 \mathrm{H}, \mathrm{CH}_{3}\right) ;{ }^{13} \mathrm{C} \mathrm{NMR}\left(101 \mathrm{MHz}, \mathrm{CDCl}_{3}\right)$ $\delta: 144.0$ (C-1'), 128.4 (C-3'), 126.7 (C-2'), 126.7 (C-4'), 96.5 (d, $J=178.3 \mathrm{~Hz}, \mathrm{C}-2), 91.1$ (d, $J=$ 169.4 Hz, C-5), 72.9 (d, $J=18.2 \mathrm{~Hz}, \mathrm{C}-6), 68.9$ (dd, $J=32.4,13.1 \mathrm{~Hz}, \mathrm{C}-3), 33.5$ (d, $J=18.9 \mathrm{~Hz}, \mathrm{C}-$ 4), $18.5\left(\mathrm{~d}, J=6.0 \mathrm{~Hz}, \mathrm{CH}_{3}\right) ;{ }^{19} \mathrm{~F} \mathrm{NMR}\left(376 \mathrm{MHz} \mathrm{CDCl}_{3}\right) \delta-183.6(\mathrm{dm}, J=43.6 \mathrm{~Hz}, \mathrm{~F}-2),-194.4$ (dd, $J=50.8,10.5 \mathrm{~Hz}, \mathrm{~F}-3) ;{ }^{19} \mathrm{~F}$ NMR (decoupled) (376 MHz, $\left.\mathrm{CDCl}_{3}\right) \delta$ : -183.6 (s, F-2, -194.4 (F-3); FTMS $\left(\mathrm{ESI}^{+}\right) \mathrm{m} / \mathrm{z}$ calcd for([M]+Na) 265.1016; found 265.1009

\section{7. (1R,2s,3S,4S,6R)-4,6-Difluoro-2-methyl-2-phenylcyclohexane-1,3-diyl bis(trifluoromethanesulfonate) (21)}

Trifluoromethanesulfonic anhydride $(3.4 \mathrm{~mL}, 20.13 \mathrm{mmol})$ was added slowly to a solution of 21 and 22, (1.22 g) in pyridine $(10 \mathrm{~mL})$ at $0^{\circ} \mathrm{C}$. After stirring for $18 \mathrm{~h}$ at room tempertaure, the reaction was quenched with a mixture of water $(50 \mathrm{~mL})$ and saturated copper sulphate solution $(2 \mathrm{~mL})$ and the product extracted into diethyl ether $(50 \mathrm{~mL}$ x 3). The combined organic extracts were dried over $\mathrm{Na}_{2} \mathrm{SO}_{4}$, and concentrated. The products was re-dissolved in a minimum amount of dichloromethane and filtered through a short silica gel pad, a product ( $2 \mathrm{~g}$ ), which was taken straight to the next stage without further purification.

A sample of $\mathbf{2 1}$ was purified over silica gel (petroleum ether/ dichloromethane 9:1) for analysis.

M.p. $=139{ }^{\circ} \mathrm{C}$ (decomposed, turned black); ${ }^{1} \mathrm{H}$ NMR $\left(400 \mathrm{MHz}, \mathrm{CDCl}_{3}\right) \delta: 7.59-7.36(\mathrm{~m}, 5 \mathrm{H}, \mathrm{H}-2$ ', 3', 4'), 5.23 (dd, $J=11.7,9.1$ Hz, 2H, H-2), 5.12-4.87 (m, 2H, H-3), 3.11-2.96 (m, 1H, H-4a), 2.292.12 (m, 1H, H-4b), 1.58 (s, 3H, $\left.\mathrm{CH}_{3}\right) ;{ }^{13} \mathrm{C}$ NMR (101 MHz, $\mathrm{CDCl}_{3}$ ) $\delta: 135.9$ (C-1'), 129.4 (C-3'), 129.2 (C-2'), 126.5 (C-4'), 117.9 (q, $J=320.2 \mathrm{~Hz}, \mathrm{CF}_{3}$ ), 89.1 (d, $\left.J=17.6 \mathrm{~Hz}, \mathrm{C}-2\right), 85.8$ (dd, $J=$ 185.3, 15.1 Hz, C-3), 47.1 (C-1), 31.9 (t, $J=21.7 \mathrm{~Hz}, \mathrm{C}-4), 12.9\left(\mathrm{CH}_{3}\right) ;{ }^{19} \mathrm{~F}$ NMR (decoupled) (376 
$\left.\mathrm{MHz}, \mathrm{CDCl}_{3}\right) \delta:-74.6\left(\mathrm{~d}, J=8.3 \mathrm{~Hz}, \mathrm{CF}_{3}\right),-187.8(\mathrm{q}, J=7.5 \mathrm{~Hz}, \mathrm{~F}-3)$; FTMS $\left(\mathrm{nESI}^{+}\right) \mathrm{m} / z$ calcd for $([\mathrm{M}]+\mathrm{H})^{+} 507.0177$; found 507.3282

$4.8((1 r, 2 R, 3 S, 5 R, 6 S)-2,3,5,6$-Tetrafluoro-1-methylcyclohexyl)benzene(12) and $((3 S, 4 R, 6 S)-3,4,6$ trifluoro-2-methylenecyclohexyl)benzene (23)

$\mathrm{Et}_{3} \mathrm{~N} .3 \mathrm{HF}(6.1 \mathrm{~mL}, 38 \mathrm{mmol})$ was added to a solution of triflates 21 and $22(2 \mathrm{~g}, 3.95 \mathrm{mmol})$ at $\mathrm{rt}$. After $48 \mathrm{~h}$ stirring at $115{ }^{\circ} \mathrm{C}$ the mixture was cooled down to room temperature and poured into aq. $\mathrm{NaHCO}_{3}(10 \%, 30 \mathrm{~mL})$. The product was then extracted into diethyl ether $(50 \mathrm{~mL} \times 3)$. The combined organic extracts were dried and the product $(1.296 \mathrm{~g})$ purified over silica gel (petroleum ether/ dichloromethane 7:3) to give $\mathbf{2 3}$ as a white crystalline solid (0.213 g, $0.87 \mathrm{mmol}, 17 \%)$ and 12 $(0.307 \mathrm{~g}, 1.34 \mathrm{mmol}, 25 \%)$ as white solid.

\section{$4.9((1 r, 2 R, 3 S, 5 R, 6 S)-2,3,5,6$-Tetrafluoro-1-methylcyclohexyl)benzene (12)}

M.p. $=143{ }^{\circ} \mathrm{C}$ (decomposed, changed colour from white to yellow); ${ }^{1} \mathrm{H}$ NMR $\left(400 \mathrm{MHz}, \mathrm{CDCl}_{3}\right) \delta$ : 7.66-7.39 (m, 5H, H-2', 3', 4'), 5.47-5.21 (dd, $J=47.5,8.9$ Hz, 2H, H-2), 5.13-4.88 (m, 2H, H-3) 2.79 (m, 1H, H-4a), 2.53 (m, 1H, H-4b); ${ }^{13} \mathrm{C} \mathrm{NMR} \mathrm{(101} \mathrm{MHz,} \mathrm{CDCl} 3$ ) $\delta: 140.8$ (C-1'), 129.0 (C-3'), 127.3 (C-4'), 125.6 (C-2'), 90.8 (dd, $J=193.1,15.2 \mathrm{~Hz}, \mathrm{C}-2), 86.0$ (dd, $J=198.7,18.2 \mathrm{~Hz}, \mathrm{C}-3$ ), $53.6(\mathrm{C}-1), 27.5(\mathrm{C}-4), 24.2\left(\mathrm{CH}_{3}\right),{ }^{19} \mathrm{~F}$ NMR $\left(376 \mathrm{MHz}, \mathrm{CDCl}_{3}\right) \delta$ : -194.6 (d, J = 47.7 Hz, F-2), 202.1 (m, F-3); ${ }^{19} \mathrm{~F}$ NMR (decoupled) (376 MHz, $\mathrm{CDCl}_{3}$ ) $\delta:-194.6$ (dd, $J=8.9,5.6 \mathrm{~Hz}, \mathrm{~F}-2$ ), -202.1 $(\mathrm{dd}, J=8.8,5.6 \mathrm{~Hz}, \mathrm{~F}-3)$; FTMS $\left(\mathrm{ESI}^{+}\right) \mathrm{m} / z$ calcd for $([\mathrm{M}]+\mathrm{Na})^{+} 269.0929$; found 269.0919

$4.10((3 S, 4 R, 6 S)-3,4,6$-Trifluoro-2-methylenecyclohexyl)benzene (23)

M.p. $=109^{\circ} \mathrm{C} ;{ }^{1} \mathrm{H}$ NMR (400 MHz, $\left.\mathrm{CDCl}_{3}\right) \delta: 7.55-7.30$ (m, 5H, H- 2', 3', 4'), 5.53 (tt, $J=1.7,0.8$ Hz, 1H , H-7a), 5.34-4.92 (m, 2H, H-3, 5), 4.90-4.83 (m, 1H, H-7b), 3.45 (d, J = 37.6 Hz, 1H, H-6), 2.84-2.67 (m, 1H, H-4a), 2.19-1.89 (m, 1H, H-4b), 1.60-1.47 (m, 1H, H-2); ${ }^{13} \mathrm{C}$ NMR (101 MHz, $\left.\mathrm{CDCl}_{3}\right) \delta: 139.3$ (d, $\left.J=14.9 \mathrm{~Hz}, \mathrm{C}-2\right), 130.6$ (d, $\left.J=5.1 \mathrm{~Hz}, \mathrm{C}^{\prime} 1^{\prime}\right), 130.0$ (d, $J=4.4 \mathrm{~Hz}, \mathrm{C}-2$ '), 128.5 (C-3'), 127.6 (C-4'), 113.5 (d, $J=10.1 \mathrm{~Hz}, \mathrm{C}-7$ ), 90.7 (dd, $J=194.4,18.9, \mathrm{C}-6), 89.7$ (d, $J=189 \mathrm{~Hz}$, C-3), 88.1 (dd, $J=184.9,20.1 \mathrm{~Hz}, \mathrm{C}-5), 51.9$ (dd, $J=19.6,2.7 \mathrm{~Hz}, \mathrm{C}-2), 33.7$ (td, $J=20.2,6.2 \mathrm{~Hz}$, C-4); ${ }^{19} \mathrm{~F}$ NMR (377 MHz, $\left.\mathrm{CDCl}_{3}\right) \delta$ : -185.4-(-186.4) (m, F-3), -194.59 (ddq, $J=45.0,12.6,6.3 \mathrm{~Hz}$, F-6), -197.7-199.6 (m, F-5); ${ }^{19} \mathrm{~F}$ NMR (decoupled) (377 MHz, $\left.\mathrm{CDCl}_{3}\right) \delta:-186.0$ (d, J=20.2 Hz, F3), $-194.6(\mathrm{~d}, J=12.9 \mathrm{~Hz}, \mathrm{~F}-6),-198.64(\mathrm{dd}, J=19.9,12.8 \mathrm{~Hz}, \mathrm{~F}-5)$; FTMS $\left(\mathrm{APCI}^{+}\right) \mathrm{m} / \mathrm{z}$ calcd for ([M]) 226.0919; found 226.0914; calcd for ([M]-H) 225.0886; found 225.0884

\section{References}

[1] A.J. Durie, A. M. Z. Slawin, T. Lebl, P. Kirsch, D. O'Hagan, Chem.Commun. 47 (2011) 82658267.

[2] A.J. Durie, A. M. Z. Slawin, T. Lebl, P. Kirsch, D. O'Hagan, Chem.Commun. 48 (2012) 96439645. 
[3] A.J. Durie, A. M. Z. Slawin, T. Lebl, D. O'Hagan, Angew. Chemie. Int. Ed., 51 (2012) 1008610088.

[4] A.J. Durie, T. Fujiwara, R. Cormanich, M. Bühl, A. M. Z. Slawin, D. O'Hagan, Chem. Eur.J. 20 (2014) 6259-6263.

[5] A.J. Durie, T. Fujiwara, N. Al-Maharik, A. M. Z. Slawin, D. O’Hagan, J. Org. Chem. 79 (2014) 8228-8233.

[6] M.S. Ayoup, D.B. Cordes, A. M. Z. Slawin, D. O'Hagan, Org. Biomol. Chem. 13 (2015) 56215624.

[7] K. Vorndran, T. Linker, Angew. Chemie Int. Ed. 42 (2003) 2489-2491.

[8] I. Usui, K. Nomura, B. Breit, Org. Lett. 13 (2011) 612-615.

[9] P.W. Rabideau, Z. Marcinow, , Org. React. (1992).

[10] N. Al-Maharik, P. Kirsch, A. M. Z. Slawin, D. O'Hagan, Tetrahedron. 70 (2014) 4626-4630

[11] N.N. Schwartz, J.H. Blumbergs, , J. Org. Chem. 29 (1964) 1976-1979.

[12] X-Ray data: Copies of the data can be obtained, free of charge, on application to CCDC, 12 Union Road, Cambridge CB2 1EZ, UK, (fax: +44 1223336033 or e-mail: deposit@ccdc.cam.ac.uk).

[13] G.A. Olah, J.T. Welch, Y.D. Vankar, M. Nojima, I. Kerekes, J.A. Olah, J. Org. Chem. 44 (1979) 3872-3881.

[14] G. Haufe, J. Fluorine Chem. 125 (2004) 875-894.

[15] D.J. Cram, J. Am. Chem. Soc. 71 (1949) 3863-3870.

[16] R. Akawie, J. Scarborough, J. Burr, J. Org. Chem. 24 (1959) 946-949.

[17] G. A. Olah, G. K. S. Prakash, Y. L. Chao, Helv. Chim. Acta. 64 (1981) 2528-2530.

[18] G. A. Olah , M. Alemayehu , A. H. Wu , O. Farooq, G. K. S. Prakash, J. Am. Chem. Soc., 114 (1992) 8042-8045.

[19] E. del Río, M.I. Menéndez, R. López, T.L. Sordo, J. Am. Chem. Soc. 123 (2001) 5064-5068.

[20] Y. Tsuji, S. Ogawa, J.P. Richard, J. Phys. Org. Chem. 26 (2013) 970-976.

[21] N.S. Keddie, A. M. Z. Slawin, T. Lebl, D. Philp, D. O'Hagan, Nature Chem., 7 (2015) 483-488. 